\title{
Appendix: Additional Contributions
}

The number of contributions to the Symposium was so high that only the review and invited talks have found place, in the form of articles, in this volume. This Appendix lists all these additional contributions (oral and posters) which are not present as articles. The abstracts of all contributions were published in a booklet produced by the Local Organizing Committee and are available at the SAO/NASA Astrophysics Data System (ADS). More information on these contributions (PowerPoint presentations and/or articles) have been made public in the Internet web site of the conference (http://cab.intacsic.es/molecular_universe/).

\section{Oral Presentations}

\section{Exoplanetary atmosphere observations}

G. Tinetti

\section{Exoplanetary atmosphere models}

E. Hebrard

\section{Galactic Center clouds and complex molecules}

J. Martín-Pintado, M.A. Torres-Requena

\section{The $\mathrm{D} / \mathrm{H}$ ratio of water ice in star formation}

Jeong-Eun Lee, Edwin Bergin

\section{Herschel introduction}

G. Pilbratt

Molecules in protostellar shocks: the CHESS view on L1157-B1

Bertrand Lefloch, M. Benedettini, S. Cabrit, E. Caux, C. Ceccarelli, J. Cernicharo, C. Codella, T. Giannini, B. Nisini, B. Parise, M. Salez, M. Vasta, S. Viti, and the CHESS team

Herschel observations of EXtra-Ordinary Sources (HEXOS): Analysis of the HIFI 1.2 THz Wide Spectral Survey toward Orion KL

Nathan R. Crockett, Edwin A. Bergin, Tom Bell, Geoffrey Blake, Jose Cernicharo, Martin Emprechtinger, Harshal Gupta, Dariusz Lis, Steven Lord, John Pearson, Rene Plume, Peter Schilke, Floris van der Tak, Shiya Wang, Shanshan Yu

\section{WISHes coming true: low-mass protostars as chemical fountains}

Lars E. Kristensen, Ewine van Dishoeck, Umut Yıldız, Ruud Visser, Greg Herczeg, Jes Jørgensen, Tim van Kempen, Michiel Hogerheijde, and the WISH team 
The chemistry of water in the ultracompact HII region (UC HII) Mon R2

Asunción Fuente, Paolo Pilleri, José Cernicharo, Olivier Berné, José Ricardo Rizzo, Manuel González-García, Javier R. Goicoechea, Volker Ossenkopf, Maryvonne Gerin, and the WADI KP team

The atmospheres of Titan and Saturn in the infrared from Cassini: the interplay between observation and laboratory studies

Donald E. Jennings, Conor A. Nixon, F. Michael Flasar, Virgil G. Kunde, Athena Coustenis

Herschel observations of comet 103P/Hartley 2: D/H in a Jupiter family comet

D. C. Lis, P. Hartogh, D. Bockelée-Morvan, M. de Val-Borro, N. Biver, M. Küppers, M. Emprechtinger, E.A. Bergin, J. Crovisier, M. Rengel, R. Moreno, G.A. Blake, S. Szutowicz

Detecting the cold water reservoir in a protoplanetary disk

Michiel Hogerheijde, Edwin Bergin, Christian Brinch, Lauren Cleeves, Jeffrey Fogel, Geoffrey Blake, José Cernicharo, Carsten Dominik, Darek Lis, Gary Melnick, David Neufeld, Olja Panić, John Pearson, Lars Kristensen, Umut Yıldız, Ewine van Dishoeck

Time-dependent anion chemistry in the CSE IRC+10216

Michel Guelin, Marce Agundez, Jose Cernicharo, Carl Gottlieb, Mike McCarthy, Patrick Thaddeus

Formation and destruction processes of carbonaceous interstellar dust Cesar S. Contreras, Claire L. Ricketts, Farid Salama

New chemical models for new era observations: a multiphase Monte Carlo model of gas-grain chemistry

Anton I. Vasyunin, Eric Herbst

Herschel oxygen project observations of molecular oxygen in Orion Paul F. Goldsmith, René Liseau, Tom Bell, Darek Lis, Jo-Hsin Chen, Ron Snell, Di Li, Michael Kaufman, Edwin Bergin, Gary Melnick, and the HOP Team

Chemical enrichment of the interstellar medium through the mass loss of evolved stars

Leen Decin, on behalf of the HIFISTARS and MESS GTKP consortia

Modelling the gas and the dust of protoplanetary disks in the Herschel-GASPS sample

Wing-Fai Thi, and the GASPS Team

Where is the molecular gas in low metallicity dwarf galaxies?

Suzanne C. Madden, Diane Cormier, Vianney Lebouteiller, SPIRE SAG 2 consortium, and PACS GT Consortium 
An interferometric 270-355 GHz spectral line survey of the red supergiant VY CMa

Karl M. Menten, Ken H. Young, Nimesh A. Patel, Carl A. Gottlieb, Patrick

Thaddeus, Michael C. McCarthy, Mark A. Gurwell, Arnaud Belloche, Tomasz

Kaminski, Lies Verheyen, Leen Decin, Sandra Bünken, Holger S. P. Müller

\section{DISCS: A Disk Imaging Survey of Chemistry with the SMA}

Edwin Bergin, Karin Öberg, Chunhua Qi, David J. Wilner, Jeffery K. J. Fogel, Sean

M. Andrews, Lauren Cleeves, Catherine Espaillat, Ilaria Pasucci

The far-ultraviolet molecular spectrum of protoplanetary disks: new views from Hubble

Kevin France

Competing mechanisms in the formation of molecular hydrogen on silicates in conditions pertinent to the ISM

J.L. Lemaire, G. Vidali, S. Baouche, M. Chehrouri,

Molecular clouds at the reionization epoch

Amiel Sternberg, Alex Dalgarno, Eric Herbst, Yezhe Pei

The chemistry of exoplanet atmospheres

Catherine Walsh, T. J. Millar

Nobeyama $45 \mathrm{~m}$ telescope legacy project: Line survey

Shuro Takano, Yuri Aikawa, Vivien Chen, Naomi Hirano, Masaaki Hiramatsu, Tomoya Hirota, Kazuhisa Kamegai, Kaori Kobayashi, Kotaro Kohno, Yi-Jehng Kuan, Sheng-Yuan Liu, Taku Nakajima, Hideko Nomura, Nagayoshi Ohashi, Masatoshi Ohishi, Hiroyuki Ozeki, Nami Sakai, Takeshi Sakai, Shoichi Shiba, Yu-Nung Su, Mika Sugimura, Shigehisa Takakuwa, Tomofumi Umemoto, Kuo-Song Wang, Masako Yamada, Takahiro Yamaguchi, Satoshi Yamamoto, Qi-Zhou Zhang

Overview of data bases

T. J. Millar

Laboratory investigations of the formation of superhydrogenated PAHs

John Thrower, Emil Friis, Bjarke Jørgensen, Louis Nilsson, Saoud Baouche, Richard Balog, Andrew Cassidy, Liv Hornekær

$\mathbf{H}_{2} \mathbf{C C C}$ - A diffuse interstellar band carrier

Ranjini Raghunandan, John P. Maier, Gordon A. H. Walker, David A. Bohlender, Fabio J. Mazzotti, Jan Fulara, Iryna Garkusha, Adam Nagy

Spectroscopy of PAHs with carbon side chains

Gaël Rouillé, Mathias Steglich, Yvain Carpentier, Friedrich Huisken, Thomas Henning

Exploring the Central Molecular Zone of the Galaxy with $\mathrm{H}_{3}^{+}$and $\mathrm{CO}$ T. R. Geballe, T. Oka, M. Goto, N. Indriolo, B. J. McCall 
Herschel/PACS detection of far-IR OH emission towards the Orion Bar PDR

J. R. Goicoechea, C. Joblin, A. Contursi, O. Berné, J. Cernicharo, M. Gerin, J. Le

Bourlot, E.A. Bergin, and the HEXOS-GT-KP team

The ubiquitous nature of $\mathbf{H F}$

T. G. Phillips, R. Monje, P. Sonnentruker

Probing the diffuse interstellar medium with hydroxyl cations and water cations

David Neufeld, David Hollenbach, Michael Kaufman, Mark Wolfire, Javier

Goicoechea, Maryvonne Gerin, and the PRISMAS team

Carbon chemistry in transitional clouds from the GOT C+ Survey of CII 158 micron emission in the Galactic Plane

William D. Langer, Thangasamy Velusamy, Jorge Pineda, Karen Willacy, Paul F. Goldsmith

Herschel observation of $\mathrm{C}_{3}$ in star forming regions

B. Mookerjea, T. Giesen, J. Stutzki, J. Cernicharo, J. Goicoechea, J. Black

\section{Posters: Session 1}

I.01. AKARI observations of ice absorption bands towards edge-on YSOs

Yuri Aikawa, Dai Kamuro, Itsuki Sakon, Yoichi Itoh, Jennifer A. Noble, Klaus M. Pontoppidan, Helen J. Fraser, Hiroshi Terada, Motohide Tamura, Ryo

Kandori, Akiko Kawamura, Munetaka Ueno

I.02. Thermal structure of a protoplanetary disk around HD163296: A study of vertical temperature distribution by $\mathrm{CO}$ emission lines

Eiji Akiyama, Munekate Momose, Hiroyuki Hayashi, Yoshimi Kitamura

I.03. Deuterium chemistry in protoplanetary disks

Tobias Albertsson, Dmitry Semenov, Thomas Henning

I.04. Complex molecules in protostellar outflows

H. Arce, J. Santiago-Garcia, D. Mardones, J. K. Jorgensen, G. Garay, M.

Tafalla, R. Bachiller

I.05. X-rays in protoplanetary disks: their impact on the thermal and chemical structure, a grid of models.

Giambattista Aresu, Inga Kamp, Rowin Meijerink, Peter Woitke, Wing Fai

Thi, Marco Spaans 
I.06. New probes of the chemistry in the inner regions of planet-forming disks

Jeanette Bast, Avi Mandell, Ewine van Dishoeck, Daniel Harsono,

Simon Bruderer

I.07. Tracing Xray and FUV radiation in the embedded phase of star formation

Arnold O. Benz, Simon Bruderer, Susanne F. Wampfler, Carolin Dedes, Ewine

F. van Dishoeck

I.08. Herschel/HIFI-HRS observation of $\mathrm{CH}$ absorption in IRAS16293-2422

Sandrine Bottinelli, Emmanuel Caux, Valentine Wakelam, Cecilia Ceccarelli, Claudine Kahane, and the CHESS team

I.09. Principles and promise of Fabry-Perot resonators at $\mathrm{THz}$ frequencies

Rogier Braakman, Geoffrey A. Blake,

I.10. A VLT-CRIRES 4.7 micron survey of CO emission from young protoplanetary disks

Joanna Brown, Klaus Pontoppidan, Ewine van Dishoeck, Gregory Herczeg

I.11. High-J CO emission in young stellar objects: Disks and Outflow walls! FUV or Shocks?

Simon Bruderer, Ewine F. van Dishoeck, S.D. Doty

I.12. New constraints on the origin of protostellar jets: The SiO abundance in $\mathrm{HH212}$

Sylvie Cabrit, Claudio Codella, Frederic Gueth, Vianney Taquet

I.13. Star forming regions towards stellar wind bubbles: the ring nebulae RCW 52 and RCW 78

Cristina Cappa, Gisela A. Romero, Mónica Rubio, M.C. Martín

I.14. Negative ions induced processes in proto-planetary atmospheres and ISM

F. Carelli, F. Sebastianelli, F. A. Gianturco

I.15. Construction of a high-resolution terahertz cavity ringdown spectrometer

P. Brandon Carroll, Brett A. McGuire, Susanna L. Widicus Weaver

I.16. The role of $\mathrm{OH}$ in the chemical evolution of protoplanetary disks

G. Chaparro, I. Kamp 
I.17. Water in massive star-forming regions with Herschel Space Observatory

Luis Chavarria, Fabrice Herpin, Sylvain Bontemps, Thierry Jacq, Alain Baudry, Jonathan Braine, Floris van de Tak, Friedrich Wyrowski, Ewine van Dishoeck

I.18. Chemistry in Very Low Luminosity Objects (VeLLOs)

Jo-Hsin Chen, Neal Evans, Jeong-Eun Lee, Michael M. Dunham, Hyo Jeong Kim

I.19. Chemical signposts in transition disks

Ilse Cleeves, Edwin Bergin, Jeffrey Fogel

I.20. The SEVO experiment onboard NASA's O/OREOS Small Satellite

Amanda Cook, Andrew Mattioda, Nathan Bramall, Kathryn Bryson, Julie

Chittenden, Pascale Ehrenfreund, Giovanni Minelli, Richard Quinn, Antonio Ricco

I.21. Organic chemistry of Cha-MMS1 and IRAS 15194-5115 probed by microwave spectroscopy

Martin Cordiner, Steven Charnley

I.22. Molecular anions in protostars, prestellar cores and dark clouds Martin Cordiner, Steven Charnley, Jane Buckle, Catherine Wash, Tom Millar

I.23. Study of deuterated water in the low-mass protostar IRAS16293-2422

Audrey Coutens, Charlotte Vastel, Emmanuel Caux, Cecilia Ceccarelli, and the CHESS team

I.24. HNC in the C-rich envelope of the AGB star IRC+10216

Fabien Daniel, Marcelino Agundez, Elvire de Beck, Jose Cernicharo, Leen Decin, Robin Lombaert

I.25. Detection of iron containing crystalline olivines in the debris disk of $\beta$ Pictoris

B.L. de Vries, B. Acke, B. Vandenbussche, L. B. F. M. Waters, J. A. D. L. Blommaert, C. Waelkens

I.26. The photodissociation of formaldehyde in comets Paul D. Feldman

I.27. The relation between deuteration and evolution in massive star formation

Francesco Fontani, Aina Palau, Paola Caselli, Alvaro Sanchez-Monge, Jonathan Tan, Michael J. Butler, Izaskun Jimenez-Serra

I.28. An improved equation of state for protoplanetary simulations

Marina Galvagni, Tristen Hayfield, Lucio Mayer, Prasenjit Saha 
I.29. On the formation of fullerenes in H-rich circumstellar environments

Anibal Garcia-Hernandez

I.30. Spitzer spectral line mapping of protostellar outflows: $\mathbf{H}_{2}$ emission in L1448, BHR71, and NGC2071

Teresa Giannini, Brunella Nisini, David Neufeld, Yuan Yuan, Simone Antoniucci, Antoine Gusdorf

I.31. New observations of molecular absorptions toward GV Tau N Erika Gibb, Matthew Troutman

I.32. SAFARI: A Far Infrared Imaging FTS-Spectrometer for SPICA J.R. Goicoechea, P.R. Roelfsema, W. Jellema, B.M. Swinyard

I.33. Molecular emission in the IC443 supernova remnant

Antoine Gusdorf, Rolf Güsten, Yuan Yuan, David Neufeld, and the WADI team

I.34. PAH Anions in circumstellar and interstellar environments Mark Hammonds, Alessandra Candian, Peter Sarre

I.35. Modelling molecular emission from young embedded disks Daniel Harsono, Ruud Visser, Ewine van Dishoeck, Lars Kristensen, Simon Bruderer, Christian Brinch, Michiel Hogerheijde

I.36. Warm water in Herschel/PACS observations of NGC 1333 IRAS 4B: the outflow, not the disk!

Gregory J. Herczeg, Agata Karska, Lars E. Kristensen, Ewine F. van Dishoeck, Ruud Visser, Jes Jorgensen, Simon Bruderer, Umut Yildiz, and the WISH Herschel Key Program Team

I.37. $\mathbf{H}_{2}$ energetics and gravitational fragmentation in the overlap region in the Antennae. "Early stages of star formation"

Cinthya Herrera, Francois Boulanger, Nicole Nesvadba

I.38. Chemical evolution of atmospheres of $\mathrm{H}_{2} \mathrm{O}$ dominant comet nuclei

Subhon Ibadov

I.39. Modelling the chemistry of a gravitationally unstable protoplanetary disc

John Ilee, Aaron Boley, Paola Caselli, Richard Durisen, Tom Hartquist, Jonathan Rawlings

I.40. Chemical segregation in hot cores: SMA imaging of the AFGL2591 star forming region

Izaskun Jimenez-Serra, Qizhou Zhang, Jesus Martin-Pintado, Serena Viti, Willem-Jan de Wit 
I.41. The structure and appearance of irradiated protoplanetary disks: the role of chemistry

Inga Kamp, Giambattista Aresu, German Chaparro, Peter Woitke, Wing-Fai Thi

I.42. Herschel/PACS observations of Class 0/I low-mass young stellar objects.

Agata Karska, Gregory Herczeg, Ewine van Dishoeck, Lars E. Kristensen, and WISH team

I.43. The search for evidence of episodic mass accretion: $\mathrm{CO}_{2}$ features in low-luminosity, embedded protostars

Hyo Jeong Kim, Neal J. Evans II, Michael M. Dunham, Jeong-Eun Lee

I.44. Epic changes in the IRS 46 mid-infrared spectrum; an inner disk chemistry study

Fred Lahuis, Inga Kamp, Wing Fai Thi, Ewine van Dishoeck,

Peter Woitke

I.45. The molecular universe as seen by JWST-MIRI

Fred Lahuis, Ewine van Dishoeck, Gillian Wright, George Rieke, and the MIRI Team

I.46. Class I methanol maser observations at $44 \mathrm{GHz}$ in the direction of some SNRs and SFRs.

G. M. Larionov, I. D. Litovchenko, I. E. Val'tts, A. V. Alakoz

I.47. OH 1720-MHz observations toward northern class I methanol masers with 70-m Ukrainian Telescope.

G. M. Larionov, I. D. Litovchenko, O. S. Bayandina, I. E. Val'tts, A. V.

Alakoz, D. V. Mukha, A. S. Nabatov, A. A. Konovalenko, V. V. Zakharenko,

E. V. Alekseev, V. S. Nikolaenko, V. F. Kulishenko, S. A. Odincov

I.48. Non-LTE infrared emission from protoplanetary disk surfaces

Alexandra Lockwood, Geoffrey Blake

I.49. PACS molecular spectroscopy of OH/IR stars

R. Lombaert, B. L. de Vries, L. Decin, J. A. D. L. Blommaert, P. Royer, E. De Beck, A. de Koter, L. B. F. M. Waters

I.50. Modeling photodissociation of $\mathrm{CO}$ isotopologues in a turbulent solar nebula

James Lyons

I.51. Emission spectra of Geminid Fireballs from 3200 Phaeton:

Preferential depleation of volatile phases

Jose Maria Madiedo, Josep Maria Trigo-Rodriguez 


\section{I.52. Water profiles of intermediate mass YSOs from HIFI}

Carolyn MCoey, Samuel Tisi, Doug Johnstone, Michel Fich, Tim A. van

Kempen, Asuncion Fuente, Paola Caselli, Jose Cernicharo, Lars E. Kristensen, Ewine F. van Dishoeck

I.53. Molecules in protoplanetary HAEBE discs as seen with Herschel.

Gwendolyn Meeus

I.54. The Herschel Orion Protostar Survey: Probing protostellar evolution with molecules, ice and dust

Tom Megeath, Manoj Puravankara, Dan Watson, Charles Poteet, Will Fischer, Babar Ali, Roland Vavrek, and the HOPS team

I.55. Centimeter, millimeter, and submillimeter observations of Comet 103P/Hartley 2

Stefanie N. Milam, Steven B. Charnley, Yo-Ling Chuang, Yi-Jehng Kuan, Iain M. Coulson, Anthony J. Remijan

I.56. Multiwavelength observations of volatiles in comets

Stefanie N. Milam, Steven B. Charnley, Yi-Jehng Kuan, Yo-Ling Chuang,

Michael A. DiSanti, Boncho P. Bonev, Anthony J. Remijan

I.57. Can PAH clusters survive in PDRs?

Julien Montillaud, Christine Joblin, Dominique Toublanc, Aude Simon, Pascal Parneix

I.58. Sulphur-bearing molecules in the ultracompact HII region G10.6-0.4

Bhaswati Mookerjea, Massimo De Luca, Tom Bell, Audrey Coutens, Maryvonne Gerin, Harshal Gupta, Raquel Monje, Charlotte Vastel

I.59. Chemical evolution of protoplanetary disks: The effects of viscous accretion, turbulent mixing, and disk winds

Hideko Nomura, Dominikus Heinzeller, Catherine Walsh, Tom Millar

I.60. Observations of near-infrared line ratios of molecular hydrogen emission to diagnose dust evolution in protoplanetary disks

Hideko Nomura, Yuhei Takagi, Yoichi Itoh, Koji Sugitani, Makoto Watanabe, Jeff Bary, David Weintraub, Yuri Aikawa, Masahiro Tsujimoto, Yoshitsugu Nakagawa, Tom Millar

I.61. Carbon fractionation in photon-dominated regions

Volker Ossenkopf, Markus Röllig, Asunción Fuente, Robert Simon, Carsten Kramer, Edwin Bergin, the whole WADI key project team, the whole HEXOS key project team

I.62. Molecular complexity in the Cep E protostellar outflow

Susana Pacheco Vazquez, Bertrand Lefloch, Cecilia Ceccarelli 
I.63. Disks, outflows, and hot cores in the $\mathrm{mm}$ range at subarcsecond angular resolution

Aina Palau, Asunción Fuente, Jérémie Boissier, Vincent Piétu, Álvaro

Sánchez-Monge, Luis Zapata, Francesco Fontani, Josep Miquel Girart, Roberto

Neri, Gemma Busquet, Qizhou Zhang, Paul T. P. Ho, Robert Estalella, Marc

Audard, Tomas Alonso-Albi

I.64. Chemical evolution in protoplanetary disks around T Tauri stars

Andrey Paska, Andrew Markwick

I.65. Origin of water around deeply embedded low-mass protostars

Magnus V. Persson, Jes K. Jørgensen, Ewine F. van Dishoeck

I.66. Production of small hydrocarbons in photo-dissociation regions

Paolo Pilleri, Christine Joblin, Maryvonne Gerin, Jerome Pety, Julien

Montillaud, Francois Boulanger, Asuncion Fuente

I.67. Herschel/PACS observations of young sources in Taurus: the far-infrared counterpart of optical jets

Linda Podio, \& the GASPS team

I.68. Carbon chemistry in oxygen rich planetary nebulae

Lizette Ramirez

I.69. Stellar winds in hot water

Anita M S Richards, Khudhair Assaf, Moshe Elitzur, Sandra Etoka, Malcolm

Gray, Graham Harper, Liz Humphreys, Jeremy Lim, Din van Trung, Jeremy Yates

I.70. Thermal emission of $\mathrm{CO}$ and isotopomers from the envelopes of water fountain stars

J. Ricardo Rizzo, J. F. Gómez, M. Osorio, O. Suárez, L. F. Miranda

I.71. The search for the magnetic precursor of C-type shocks in young molecular outflows

Julia Roberts, Izaskun Jiménez-Serra, Jesus Martín-Pintado

I.72. Radio spectroscopy of circumstellar molecular masers

Georgij M. Rudnitskij, Pierre Colom, Evguenij E. Lekht, Mikhail I.

Pashchenko, Vladimir A. Samodurov, Il'nur A. Surbaev, Alexander M.

Tolmachev

I.73. Deuterium fractionation in massive clumps in early evolutionary stages of high-mass star formation

Takeshi Sakai, Nami Sakai, Kenji Furuya, Yuri Aikawa, Tomoya Hirota, Satoshi Yamamoto 
I.74. Linking high-J CO emission from low- to high-mass protostars with Herschel-HIFI

Irene San José-García, Lars E. Kristensen, Umut A. Yıldız, Ewine F. van

Dishoeck, and the WISH team

I.75. Molecular complexity in O-rich circumstellar envelopes around evolved stars: IK TAU and $\mathrm{OH} 231.8+4.2$

C. Sánchez Contreras, L. Velilla Prieto, J. Cernicharo, J. Alcolea, J.R. Pardo,

M. Agúndez, V. Bujarrabal, F. Herpin, K.M. Menten, F. Wyroswky

I.76. Hot molecular cores in young stellar objects of different luminosities

Álvaro Sánchez-Monge, Aina Palau, Robert Estalella, Stan Kurtz

I.77. Observing water in low-mass proto-stellar outflows: the case of L1448

Gina Santangelo, Brunella Nisini, Simone Antoniucci, Teresa Giannini, Milena Benedettini, Claudio Codella, René Liseau, Andrea Lorenzani, Mario Tafalla, Magda Vasta, Ewine van Dishoeck, Lars Kristensen

I.78. Characterizing $\mathrm{CO}$ fourth positive emission in proto-planetary disks

Eric Schindhelm, Kevin France, Eric Burgh, Alex Brown, Greg Herczeg

I.79. Cool molecular environment of stellar mergers V838 Mon and V4332 Sgr. Observations of the circumstellar ammonia $1_{0}-0_{0}$ lines in carbon-rich AGB stars by the Herschel/HIFI

Mirosław R. Schmidt, Romuald Tylenda, Tomasz Kamiński, M.R. Schmidt, D. A. Neufeld, R. Szczerba, J. He, N. Siódmiak, and the HIFISTARS consortium

I.80. Effect of metallicity on the chemical properties of ices around embedded young stellar objects

Takashi Shimonishi, Takashi Onaka, Daisuke Kato, Yoshifusa Ita, Itsuki Sakon, Akiko Kawamura, Hidehiro Kaneda

I.81. Spectroscopic analysis of cool galactic R CrB Star candidates Olesja Smirnova, Laimons Začs, Yakiv V. Pavlenko, Bogdan Kaminsky

I.82. Observational signatures of ${ }^{12} \mathrm{CO}-{ }^{13} \mathrm{CO}$ partitioning in ice and gas towards local young stellar objects and molecular clouds

Rachel L. Smith, Klaus M. Pontoppidan, Edward D. Young, Mark R. Morris

I.83. Cosmic origins spectrograph observations of dense translucent clouds

Theodore P. Snow, E. B. Burgh, J. P. Destree

I.84. Periodic outbursts in the $6.7 \mathrm{GHz}$ methanol maser line towards G22.356+0.066

M. Szymczak, P. Wolak, A. Bartkiewicz 
I.85. A molecular survey of outflow gas: velocity-dependent shock chemistry and the peculiar composition of the extremely high-velocity (EHV) gas

Mario Tafalla, Joaquín Santiago-García, Alvaro Hacar, Rafael Bachiller

I.86. Molecular chemistry as a probe of pre-stellar core dynamics Konstantinos Tassis, Karen Willacy, Harold Yorke, Neal Turner

I.87. Herschel/HIFI view on massive evolved stars: the HIFISTARS sample of supergiant and yellow hypergiant envelopes

David Teyssier, Anthony Marston, Javier Alcolea, Valentin Bujarrabal, and the HIFISTARS consortium

I.88. Modelling $\mathrm{CH}^{+}$in the protoplanetary disk HD100546

Wing-Fai Thi, F. Ménard, G. Meeus, C. Martin-Zaïdi, P. Woitke, E. Tatulli, M. Benisty, I. Kamp, I. Pascucci, C. Pinte, C. A. Grady, S. Brittain, G.J. White, C. D. Howard, G. Sandell, C. Eiroa

I.89. Modelling the gas and dust of protoplanetary disks in the Herschel-GASPS sample

Wing-Fai Thi, and the GASPS Team

I.90. Low-temperature kinetic studies of $\mathrm{OH}$ radical reactions relevant to planetary atmospheres

Thomas M. Townsend, Maria Antiñolo, Bernabe Ballesteros, Elena Jimenez, Andre Canosa

I.91. The pathways of C: from AGB stars, to the Interstellar Medium, and finally into the protoplanetary disk

Josep M. Trigo-Rodriguez, D. Anibal Garcia-Hernandez

I.92. The extent of aqueous alteration in C-class asteroids, and the survival of presolar isotopic signatures in chondrites Josep M. Trigo-Rodriguez

I.93. Searching for a spectro-astrometric signal from molecules in circumstellar disks

Matthew Troutman, Erika Gibb

I.94. Complex organic molecules toward low-mass protostars and outflows

Nienke van der Marel, Karin I. Öberg, Lars Kristensen, Ewine F. van Dishoeck

I.95. Herschel spectroscopy of planetary nebulae

P.A.M. van Hoof, M.J. Barlow, K.M. Exter, R. Wesson, G.C. Van de Steene,

M.A.T. Groenewegen

I.96. L1157-B1: water as a unique tracer of low velocity shocks

Serena Viti, I. Jimenez-Serra, J. Yates, C. Codella, M. Vasta, P. Caselli, B. Lefloch, C. Ceccarelli 
I.97. Herschel HIFI observation of $\mathrm{HCl}$ emission in the low mass protostar IRAS16293-2422

Valentine Wakelam, Emmanuel Caux, Sandrine Bottinelli, Cecilia Ceccarelli, Claudine Kahane, and the CHESS team

I.98. An expanding disk around the young massive star AFGL 2591 Kuo-Song Wang, Floris van der Tak, Michiel Hogerheijde

I.99. Spectral line survey toward RCrA IRS7B in the $345 \mathrm{GHz}$ window with ASTE

Yoshimasa Watanabe, Nami Sakai, Johan Lindberg, Jes Jørgensen, Suzanne Bisschop, Satoshi Yamamoto

I.100. The ${ }^{12} \mathrm{C} /{ }^{13} \mathrm{C}$ ratio as a chemistry indicator Eva Wirström, Wolf Geppert, Carina Persson, Steven Charnley

I.101. Sub-mm spectroscopy and infrared interferometry of molecular layers of evolved stars

Markus Wittkowski, E. M. L. Humphreys, M. D. Gray, D. A. Boboltz, C. de Breuck

I.102. High-J CO survey of low-mass protostars observed with Herschel-HIFI

Umut A. Yıldız, Lars E. Kristensen, Ewine F. van Dishoeck, Jes K. Jørgensen, Ruud Visser, Irene San José-García, and the WISH Team

I.103. Molecular chemistry in protostellar disk winds and observational predictions for Herschel and ALMA

W. Yvart, S. Cabrit, G. Pineau des Forêts, P.J.V. Garcia, J. Ferreira, F. Casse

I.104. Molecules in the atmosphere and circumstellar shell of proto-planetary nebula IRAS22272+5435

Laimons Zacs, Aija Laure, Julius Sperauskas

\section{Posters: Session 2}

II.01. The effects of grain size and grain growth on the chemical evolution of cold dense clouds

Kinsuk Acharyya, Eric Herbst

II.02. Revisited nitrogen isotopic ratio in molecular clouds Gilles Adande, Lucy Ziurys

II.03. Astronomical identification of $\mathrm{CN}^{-}$, the smallest observed molecular anion

Marcelino Agúndez, José Cernicharo, Michel Guélin, Claudine Kahane, E. Roueff, Jacek Kłos, Francisco Javier Aoiz, François Lique, Nuria Marcelino, Javier R. Goicoechea, Manuel González García, Carl A. Gottlieb, Mike C. McCarthy, Patrick Thaddeus 
II.04. Photoionization models of the $\mathbf{H}_{2}$ emission of the Narrow Line Region of AGNs

Isabel Aleman, Ruth Gruenwald

II.05. SO and $\mathrm{SO}_{2}$ line observations and chemical evolution of Orion KL

Gisela B. Esplugues, José Cernicharo, Serena Viti, Belén Tercero, Nuria Marcelino, Javier R. Goicioechea

II.06. Probing the physical conditions in Orion KL with methyl cyanide

T. A. Bell, J. Cernicharo, and the HEXOS team

II.07. Exciting Results from the HEXOS Herschel GT Key Program Edwin A. Bergin, and the HEXOS team

II.08. Herschel insights into the evolution of very small dust particles and the heating of interstellar gas

O. Berné, C. Joblin, P. Pilleri, J. Goicoechea, F. Salgado, Y. Okada, M. Gerin, V. Ossenkopf, E. Bergin

II.09. The molecular gas rich galaxy NGC 3934: a multiwavelenght view

Daniela Bettoni, Giuseppe Galletta, Roberto Rampazzo, Antonietta Marino, Paola Mazzei, Lucio Buson

II.10. Nitrogen isotopic fractionation in cold quiescent clouds: first results for $\mathrm{L} 1544$

Luca Bizzocchi, Paola Caselli, Luca Dore

II.11. DR21 $(\mathrm{OH})$ - a high-mass star cluster in formation observed with Herschel-HIFI, and the IRAM PdBI

S. Bontemps, T. Csengeri, F. Herpin, N. Schneider, F. Motte, L. Chavarria, A. Baudry

II.12. On the relative abundance of $\mathrm{LiH}$ and $\mathrm{LiH}+$ molecules in the early universe: new results from quantum reactions

Stefano Bovino, Mario Tacconi, Franco Gianturco

II.13. Dense gas and star formation along the major axis of M33 (HERM33ES)

Christof Buchbender, Carsten Kramer, Erik Rosolowsky

II.14. On the behavior of the $\mathrm{NH}_{3} / \mathrm{N}_{2} \mathrm{H}+$ ratio in high-mass star forming regions

Gemma Busquet, Robert Estalella, Aina Palau, Qizhou Zhang, Paul T.P. Ho, Serena Viti 
II.15. Analysis of velocity components along the line of side towards $\operatorname{SgrB2}(\mathrm{M})$

Denis Büchel, Peter Schilke, Claudia Comito, Sheng Li Qin, Edwin A Bergin, Darek C Lis

II.16. Molecular gas and star formation in Sh2-196 and Sh2-206 Cristina Cappa, M.C. Martín, S. Cichowolski, Javier Vasquez, Ricardo Zinn

II.17. Water observations with Herschel/HIFI toward AFGL 2591 Yunhee Choi, Floris van der Tak, Ewine van Dishoeck

II.18. The water content of Sgr B2(M) Claudia Comito, Peter Schilke, Darek Lis, Edwin Bergin, et al.

II.19. Radiative cooling functions for primordial molecules Carla Maria Coppola, Lorenzo Lodi, Jonathan Tennyson

II.20. Vibrational level population of $\mathbf{H}_{2}$ and $\mathbf{H}_{2}^{+}$in the early Universe

Carla Maria Coppola, Savino Longo, Mario Capitelli, Francesco Palla, Daniele Galli

II.21. Diffuse interstellar bands in the Andromeda and Triangulum galaxies

Martin Cordiner, Nick Cox, Keith Smith, Christopher Evans, Carrie Trundle, Karl Gordon, Peter Sarre

II.22. Gas properties in dwarf galaxies from Herschel FIR spectroscopic observations

Diane Cormier, Suzanne Madden, Vianney Lebouteiller, Frederic Galliano, Sacha Hony

II.23. A mm spectral survey of the Orion Bar Photo Dissociation Region

J. R. Goicoechea, S. Cuadrado, A. Fuente, G. B. Esplugues, J. Cernicharo, S. García-Burillo, A. Usero, N. Marcelino,

II.24. The molecular UCHII region W31C seen by Herschel-HIFI Massimo De Luca, Tom Bell, Audrey Coutens, Maryvonne Gerin, Harshal Gupta, Raquel Monje, Bhaswati Mookerjea, Charlotte Vastel

II.25. Studying cooling mechanisms in the massive star forming region IRAS 12326-6245

Carolin Dedes, Fabrice Herpin, Luis Chavarria, Susanne Wampfler, Friedrich Wyrowski, Floris van der Tak, Arnold Benz, Simon Bruderer, Edward Polehampton, Martin Melchior

II.26. Large area mappings of formaldehyde at 6-cm toward Giant Molecular Clouds

Jarken Esimbek, Jianjun Zhou, Chuanpeng Zhang 
II.27. Gas and dust in the Galactic Centre - ISO LWS, submillimetre line and continuum observations.

Mireya Etxaluze, Glenn J. White, Howard A. Smith, Eduardo

Gonzalez-Alfonso, Antony A. Stark, Gordon J. Stacey, Sarah J. Leeks, Ian

Gatley, Jacqueline Fisher, Douglas Pierce-Price, John S. Richer, Tim W.

Grundy, Ed T. Polehampton

II.28. $\mathrm{HCOOCH}_{3}$ as a probe of temperature and structure of Orion-KL

Cecile Favre, Didier Despois, Nathalie Brouillet, Alain Baudry, Francoise

Combes, Michel Guelin, Al Wootten, Georges Wlodarczak

II.29. NGC4945 and NGC 253 line survey

Paola Fiadino, Julia Roberts

II.30. Water in the Milky Way: Absorption lines along the PRISMAS lines of sight

Nicolas Flagey, Paul Goldsmith, David Neufeld, Darek Lis, and the PRISMAS team

II.31. Chemical differentiation in magnetized young starless cores in the Pipe Nebula

Pau Frau, Josep M. Girart, Maria T. Beltrán, Óscar Morata

II.32. Carbon Isotope and Isotopomer Fractionation in Dense Molecular Cloud Cores

Kenji Furuya, Yuri Aikawa, Nami Sakai, Satoshi Yamamoto

II.33. Molecular evolution of a first core in 3 dimensional hydrodynamic Calculations

Kenji Furuya, Yuri Aikawa, Tomoaki Matsumoto, Kengo Tomida, Kazuya

Saigo, Kohji Tomisaka, Franck Hersant, Valentine Wakelam

II.34. Dense molecular gas tracers and star formation rate in galaxies near and far

$\mathrm{Yu}$ Gao

II.35. Large-scale molecular shocks in galaxies: the imprint of mechanical dominated regions (MDR)

Santiago Garcia-Burillo, Antonio Usero, Asuncion Fuente, Javier

Gracia-Carpio, Andrew J Baker, Jesus Martin-Pintado

II.36. Formation of carbonaceous molecules in the ISM

Thomas F. Giesen, Volker Lutter, Imke Gottbehüt, Jürgen Krieg, Christian

Endres, Sven Thorwirth, Stephan Schlemmer

II.37. Tunnelling in space

T.P.M. Goumans, S. Andersson, J. Kaestner 
II.38. The Horsehead Nebula: a template for extragalactic high density tracers studies?

Pierre Gratier, Jérôme Pety, Maryvonne Gerin, Julien Montillaud, Viviana Guzmán, Javier R. Goicoechea,

II.39. Detection of $\mathrm{OH}^{+}$and $\mathrm{H}_{2} \mathrm{O}^{+}$towards Orion $\mathrm{KL}$

Harshal Gupta, Paul Rimmer, John C. Pearson, Eric Herbst, Shanshan Yu, Edwin A. Bergin, and the HEXOS team

II.40. $\mathrm{H}_{2} \mathrm{CO}$ in the Horsehead nebula

Viviana Guzmán, Jerôme Pety, Javier R. Goicoechea, Maryvonne Gerin, Evelyne Roueff

II.41. Physical conditions and chemical evolution of the gas towards the Orion Bar photodissociation region using Herschel/PACS and SPIRE spectro-imaging observations

Emilie Habart, Jeronimo Bernard-Salas, Emmanuel Dartois, Heddy Arab, Alain Abergel, and the SPIRE consortium

II.42. Model of molecular abundance in an AGN disk of NGC 1068 Nanase Harada, Todd Thompson, Eric Herbst

II.43. Physical/chemical structure and kinematics of an extremely filamentary Infrared Dark Cloud

Jonathan D. Henshaw, Paola Caselli, Izaskun Jiménez-Serra, Francesco

Fontani, Jonathan Tan

II.44. Oxygen depletion in dense molecular clouds: a clue to a low $\mathrm{O}_{2}$ abundance?

Ugo Hincelin, Valentine Wakelam, Franck Hersant, Stéphane Guilloteau, Jean-Christophe Loison, Pascal Honvault, Jurgen Troe

II.45. PAHs in the LMC and comparison to other galaxies Hony and SAGE-Spec team.

II.46. Spitzer spectroscopy of young stellar object candidates in the central molecular zone - Determing an average star formation rate for the center of our Galaxy

Katharina Immer, Frederic Schuller, Karl M. Menten

II.47. Spectral survey of the star-forming region W51 e1/e2 in the 3-mm wavelength range

Sergei Kalenskii, Lars E. B. Johansson

II.48. The effects of subsurface chemistry in the grain mantles on the deuterium chemistry in the molecular clouds

Juris Kalvans, Ivar Shmeld 
II.49. The Production of $\mathrm{O}_{2}$ in molecular shocks: HOP observations of Orion $\mathrm{H}_{2}$ Peak 1

Michael Kaufman, Paul Goldsmith, and the HOP team

II.50. Barely detectable molecules in translucent interstellar clouds Jacek Krełowski, Maja Kaźmierczak

II.51. Investigation of dust properties within the Ophiuchus region with multi-band observations

Dae-Hee Lee, Woong-Seob Jeong, Jeonghyun Pyo, Kwang-Il Seon

II.52. Turbulent formation and excitation of molecules in diffuse media.

Pierre Lesaffre, Benjamin Godard, Guillaume Pineau des Forêts, François Boulanger, Édith Falgarone, Maryvonne Gerin, Pierre Guillard

II.53. The distribution of warm gas in the G327.3-0.6 star forming region

Silvia Leurini, Friedrich Wyrowski, Floris van der Tak, Fabrice Herpin, and the WISH team

II.54. The impact of strong radiation fields on protostars in the $\mathbf{R}$ CrA region Johan E. Lindberg, Jes K. Jørgensen, and the DIGIT team

II.55. A Molecular line survey of IRDC star forming clumps with the Nobeyama 45M telescope

Sheng-Yuan Liu, Yu-Nung Su, and the NRO 45 m survey team

II.56. Star formation and black hole accretion in nearby (U)LIRGs. Edo Loenen, Paul van der Werf, Rowin Meijerink, and the HerCULES consortium

II.57. $\mathrm{o}-\mathrm{H}_{2}, \mathbf{p}-\mathrm{H}_{2}$ and $\mathrm{HD}$ vibrational kinetics in the Early Universe S. Longo, R. D'Introno, A. Panarese, C. M. Coppola, J. Tennyson

II.58. SiO outflows in high-mass star forming regions: a potential chemical clock

Ana López-Sepulcre, Malcolm Walmsley, Riccardo Cesaroni, Claudio Codella, Frédéric Schuller, Leonardo Bronfman, Sean J. Carey, Karl Menten, Sergio Molinari, Alberto Noriega-Crespo

II.59. Investigating the molecular content of lensed submillimeter galaxies

Roxana Lupu, and the Z-Spec team

II.60. Large scale CO emission in Orion A: Star formation feedback on the molecular gas

Nuria Marcelino, Olivier Berné, José Cernicharo 
II.61. Warm deuteration of hydrogen cyanide in Orion

Nuria Marcelino, Belén Tercero, José Cernicharo, Evelyne Roueff, Aina Palau, Javier R. Goicoechea, Edwin Bergin, and the HEXOS team

II.62. Hot Core modelling with stochastic grain surface modelling Daniel McElroy, Tom Millar

II.63. Observations of nitrogen isotope fractionation in prestellar cores

Stefanie N. Milam

II.64. Can we trust CO emission as a probe of the densities and temperatures of molecular clouds?

Faviola Molina, Simon Glover, Christoph Federrath

II.65. Herschel/HIFI observations of hydrogen fluoride along a strip towards Sagittarius B2 (M)

Raquel R. Monje, Darek Lis, Paul F. Goldsmith, Thomas G. Phillipas, David Neufeld

II.66. Dense molecular gas in starburst galaxies: Warmer than expected?

Stefanie Mühle, Christian Henkel, Tahlia de Maio, Ernie R. Seaquist

II.67. The quest for complex molecules in space. Searches for cyanides related to $n$-propyl cyanide in $\mathrm{Sgr}$ B2(N)

Holger S. P. Müller, Arnaud Belloche, Karl M. Menten, Audrey Coutens, Adam Walters, Jens-Uwe Grabow, Stephan Schlemmer

II.68. Orion S: a test for Oxygen chemistry

Zsófia Nagy, Floris van der Tak, Gary Melnick, Paul Goldsmith, Rene Plume, Edwin Bergin, Volker Tolls, and the HEXOS and HOP teams

II.69. Line survey project of external galaxies with NRO 45-m Telescope

Tac Nakajima, Shuro Takano, Kotaro Kohno, Hirofumi Inoue, and the NRO 45-m survey team

II.70. Methyl formate in star forming regions

A. Occhiogrosso, S. Viti, P. Modica, M.E. Palumbo

II.71. Nitrogen-bearing molecules in prestellar cores

Marco Padovani, Malcolm Walmsley, Mario Tafalla, Pierre Hily-Blant

II.72. ortho- $\mathrm{H}_{2}$ and the age of dark clouds and prestellar cores Laurent Pagani, Pierre Lesaffre, Pascal Honvault, Alexandre Faure, Mohammad Jorfi, Tomas Gonzales-Lezana

II.73. A Comprehensive survey of hydrogen chloride in the galaxy Ruisheng Peng, Hiroshige Yoshida, Richard A. Chamberlin, Thomas G. Phillips, Dariusz C. Lis, Maryvonne Gerin 
II.74. Acetone in Orion: high-resolution images of a special oxygen-bearing molecule

Tzu-Cheng Peng, Nathalie Brouillet, Cécile Favre, Dider Despois, Alain

Baudry, Anthony Remijan, Tom Wilson, Alwyn Wootten

II.75. Structure and dynamics of an AGN torus: 3D hydrodynamical simulations for ALMA

J.P. Perez-Beaupuits, K. Wada, M. Spaans, R. Guesten

II.76. Nitrogen hydrides in interstellar gas towards G10.6-0.4 (W31C) and W49N

C. M. Persson, M. De Luca, B. Mookerjea, M. Gerin, J. H. Black, T. A. Bell, B. Godard, J. Goicoechea, G. Hassel, E. Herbst, P. Hily-Blant, K. M. Menten, H. S. P. Müller, A. O. H Olofsson, J. C. Pearson, S. Yu, and the PRISMAS team

II.77. The Role of environment in star formation: Young clusters forming in isolation

Dawn Peterson, Rob Gutermuth, Tyler Bourke

II.78. CO (1-0) mapping of local interacting Luminous Infrared Galaxies

Andreea Petric, Joe Mazzarella, Lee Armus, Jason Surace, Jin Koda, Aaron Evans, Dave Frayer

II.79. Physical condition of molecular gas at the centre of the active galaxy NGC 1097

Nuria Piñol Ferrer, Kambiz Fathi, Andreas Lundgren, Glenn van de Ven

II.80. Probing ISM dust through X-RAY spectroscopy

Ciro Pinto, Jelle S. Kaastra, Elisa Costantini, F. Verbunt

II.81. A Direct determination of the $\mathrm{C} 18 \mathrm{O}$ column density in Orion KL

Rene Plume, E. A. Bergin, T. G. Phillips, D. C. Lis, and the HEXOS team

II.82. A High-resolution study of the near-infrared diffuse interstellar bands

Mark Rawlings, Andy Adamson, Ben McCall, Tom Kerr

II.83. The APEX spectral line surveys, more and more excited Miguel Angel Requena Torres, Rolf Güsten, Jesus Martín-Pintado, Sergio Martín, Rebeca Aladro, Axel Weiss, Bernd Klein, Stephan Heyminck

II.84. Three possible explanations for transitional molecules in Orion KL

Paul Rimmer, Eric Herbst 
II.85. The overall systematic trends in the kinematics of massive star forming regions. Observations of $\mathrm{HC}_{3} \mathrm{~N}^{*}$ in hot cores

Víctor M. Rivilla, Jesús Martín-Pintado, Izaskun Jiménez-Serra, Pablo de Vicente

II.86. A search for PAHs in the ISM: High-resolution UV observations confronted with laboratory spectra

Gaël Rouillé, Roland Gredel, Yvain Carpentier, Mathias Steglich, Friedrich Huisken, Thomas Henning

II.87. Herschel-SPIRE spectroscopy of nearby Seyfert galaxies Nicola Sacchi, and the SPIRE Sag2 consortium

II.88. Polycyclic aromatic hydrocarbons and the diffuse interstellar bands. A survey

Farid Salama, Gazinur Galazutdinov, Jacek Krelowski, Ludovic Biennier, Yuri Beletsky, In-Ok Song

II.89. Polycyclic aromatic hydrocarbon and emission line ratios in Active Galactic Nuclei and Starburst Galaxies

Dinalva A. Sales, Miriani Pastoriza, Rogério Riffel

II.90. Global collapse of the DR21 filament

N. Schneider, T. Csengeri, S. Bontemps, F. Motte, R. Simon, P. Hennebelle, C. Federrath, R. Klessen

II.91. Chemical and dynamical evolution of infrared dark clouds, massive protostars and proto star clusters

Jonathan Tan, Audra Hernandez, Michael Butler, Bo Ma, Yichen Zhang, Peter Barnes, Stefan O'Dougherty, Paola Caselli, Francesco Fontani, Izaskun Jimenez-Serra

II.92. A new model for the formation of grain mantles in Prestellar Cores

Vianney Taquet, Cecilia Ceccarelli, Claudine Kahane

II.93. A line confusion limited millimeter survey of Orion KL

B. Tercero, J. Cernicharo, J. R. Pardo, J. R. Goicoechea

II.94. Herschel observations of extra-ordinary sources: Sulfur carbon chains and silicon bearing species in Orion KL

B. Tercero, J. Cernicharo, N. Marcelino, and the HEXOS team

II.95. A multispecies survey of the active galaxy NGC1068

Antonio Usero, Santiago García-Burillo, Asunción Fuente, Susanne Aalto, Roberto Neri, Melanie Krips 
II.96. Water emission towards the chemical rich outflow L1157: the WISH spectral line survey.

Magda Vasta, Claudio Codella, Andrea Lorenzani, Gina Santangelo, Brunella Nisini, Teresa Giannini, Mario Tafalla, Rene Liseau, Lars Kristensen, Edwine van Dischoeck

II.97. Chemical composition of infrared dark clouds: observations vs modeling

Tatiana Vasyunina, Eric Herbst, Hendrik Linz, Thomas Henning, Igor Zinchenko, Henrik Beuther, Maxim Voronkov

II.98. $\mathrm{C}+/ \mathrm{CO}$ transitions in the diffuse ISM: Transitional Cloud Sample from the GOT C+ Survey of [CII] in the inner Galaxy at $1=-30$ to 30

T. Velusamy, J. L. Pineda, W. D. Langer, K. Willacy, P. F. Goldsmith

II.99. Prestellar cores with ALMA

Malcolm Walmsley

II.100. First hyperfine structure resolved OH FIR spectrum of a star-forming region

S. F. Wampfler, S. Bruderer, L. E. Kristensen, E. A. Bergin, A. O. Benz, E. F. van Dishoeck, G. J. Herczeg, F. F. S. van der Tak, J. R. Goicoechea, S. D.

Doty, F. Herpin

II.101. Chemical and thermal structure of infrared dark cloud cores Dmitri Wiebe, Yaroslav Pavlyuchenkov

II.102. The first Herschel FTS look of the M83 FIR spectrum Ronin Wu, Suzanne Madden, Pasquale Panuzzo, and the Herschel SAG2 Very Nearby Galaxy team

II.103. APEX telescope observations of hydrides

Friedrich Wyrowski, Karl Menten, Rolf Güsten, Arnaud Belloche, Thomas Klein, Christian Leinz

II.104. $\mathrm{CO}(3-2), \mathrm{CO}(2-1)$ and $\mathrm{CO}(1-0)$ observations toward the Galactic bubbles N54, N73, N74, N82 and N130

Jian-jun Zhou, Jarken Esimbek, Wei-guang Ji

II.105. Structure and chemistry of the high mass star forming region S255 on small scales

Igor Zinchenko, Stan Kurtz, Sheng-Yuan Liu, Devendra Ojha, Yu-Nung Su

II.106. A search for $\mathrm{HeH}+$ and $\mathrm{CH}$ in a high redshift QSO

Igor Zinchenko, Victor Dubrovich, Christian Henkel 


\section{Posters: Session 3}

III.01. A triplet ground state for cationic polyaromatic hydrocarbons after hydrogen loss

Hector Alvaro Galue, Jos Oomens

III.02. Photolysis and Radiolysis of Formic Acid: Relevances to Astrophysical Ices.

Diana Andrade, Alicja Domaracka, Enio Frota da Silveira, Herman Rothard, Philippe Boduc

III.03. Molecular dynamics simulations of $\mathrm{CO}_{2}$ formation in interstellar ices

Carina Arasa, Stefan Andersson, Ewine F. van Dishoeck, Geert-Jan Kroes

III.04. Molecular dynamics simulations of $\mathrm{D}_{2} \mathrm{O}$ ice photodesorption Carina Arasa, Stefan Andersson, Herma Cuppen, Ewine F. van Dishoeck, Geert-Jan Kroes

III.05. Interstellar chemistry of atomic nitrogen: low temperature kinetics of the $\mathrm{N}+\mathrm{OH}$ and $\mathrm{N}+\mathrm{NO}$ reactions.

Astrid Bergeat, Julien Daranlot, Kevin M. Hickson

III.06. Differential adsorption of complex organic molecules isomers at interstellar ices surfaces

Mathieu Bertin, Marie Lattelais, Hakima Mokrane, Françoise Pauzat, Jerôme Pilmé, Claire Minot, Yves Ellinger, Claire Romanzin, Xavier Michaut, Pascal Jeseck, Jean-Hugues Fillion, Henda Chaabouni, Emanuele Congiu, François Dulieu, Saoud Baouche, Jean-Louis Lemaire

III.07. Gas phase chemical kinetics at high temperature of carbonaceous molecules: application to circumstellar envelopes

Ludovic Biennier, Aline Gardez, Ghassen Saidani, Robert Georges, Bertrand Rowe, K.P.J. Reddy

III.08. A new geometry-free astrochemistry code for treating HII/PDR complexes

Thomas G. Bisbas, Tom A. Bell, Serena Viti, Jeremy Yates, Mike Barlow, Barbara Ercolano

III.09. Studies of sulphur containing model interstellar ices

Wendy A. Brown, Daren J. Burke, John L. Edridge

III.10. Analyzing astronomical observations with the NASA Ames PAH database.

Jan Cami, Christiaan Boersma, Els Peeters, Charles Bauschlicher Jr., Douglas Hudgins, Louis Allamandola 
III.11. Emission model of AIBs: the case of the $11.2 \lambda \mathrm{m}$ band Alessandra Candian, Peter Sarre

III.12. Observing PAH hydrogenation with scanning tunneling microscopy

Andrew M. Cassidy, Louis Nilsson, Richard Balog, John Thrower, Bjarke Jørgensen, Liv Hornekær

III.13. Grain surface polarization: ferroelectric mantles?

Andrew M. Cassidy, Richard Balog, David Field, Nykola Jones, Oksana Plekan

III.14. CASSIS, a software package to analyse high spectral resolution observations

Emmanuel Caux, Sandrine Bottinelli, Charlotte Vastel, Jean-Michel Glorian

III.15. A unified Macro Monte Carlo Simulation of gas phase chemistry and micro Monte Carlo simulation of grain surface chemistry

Qiang Chang, Eric Herbst

III.16. Stochastic models of molecule formation on dust Steven Charnley, Eva Wirstroem

III.17. Ice, dust and extinction in dense clouds Jean Chiar, Adwin Boogert, Caltech, Claudia Knez, Xander Tielens

III.18. Low temperature FIR and submm opacity of interstellar silicate dust analogs: experimental variation of the emissivity spectral index with the wavelength and temperature Anne Coupeaud, Karine Demyk, Claude Mény, Céline Nayral

III.19. Nuclear spin dependence of hydrogenic plasmas in the laboratory and the diffuse interstellar medium

Kyle N. Crabtree, Nick Indriolo, Holger Kreckel, Carrie A. Kauffman, Brian A. Tom, Eftalda Becka, Brett A. McGuire, Benjamin J. McCall,

III.20. Photodesorption and product formation in UV-irradiated ices under ultra-high-vacuum conditions.

Gustavo A. Cruz Díaz, Antonio Jiménez Escobar, Guillermo M. Muñoz Caro

III.21. Spectroscopic networks

Attila G. Császár, Tibor Furtenbacher

III.22. Simulations of grain surface chemistry under interstellar conditions

Herma Cuppen

III.23. UV induced chemistry of polycyclic aromatic hydrocarbons in $\mathrm{NH}_{3}$-containing interstellar ice analogues

Cuylle, S.H., Tenenbaum, E.D., Bouwman, J., Linnartz, H. 
III.24. Tentative detection of $\mathrm{HCl}^{+}$in diffuse clouds Massimo De Luca, Harshal Gupta, David Neufeld, Maryvonne Gerin, David Teyssier, John Pearson, Darek Lis, and the PRISMAS team

III.25. Electronic absorption spectra of diacetylene cation and comparison with diffuse interstellar bands

Rainer Dietsche, Satrajit Chakrabarty, Corey A. Rice, Fabio J. Mazzotti, John P. Maier

III.26. Formation of complex organics by gas phase and intracluster ion-molecule reactions involving acetylene and hydrogen cyanide

Samy El-Shall, Ahmed Hamed, Abdel-Rahman Soliman, Paul O. Momoh

III.27. VAMDC: A new home for molecular data for astronomy Christian Endres, Thomas Giesen, Holger Müller, Peter Schilke, Jürgen Stutzki, Stephan Schlemmer

III.28. Electronic absorption spectra of small carbon chain oxides in $6 \mathrm{~K}$ neon matrices.

Sonia M. Erattupuzha Joseph, Jan Fulara, Adam Nagy, Iryna Garkusha, John P. Maier

III.29. Photodesorption of interstellar ice analogues: a wavelength-dependent study

Edith Fayolle, Mathieu Bertin, Claire Romanzin, Xavier Michaut, Karin

Öberg, Harold Linnartz, Jean-Hugues Fillion

III.30. The chemistry of high mass star forming regions with "Chemical Differentiation": Orion KL, W75N, \& W3

Douglas N. Friedel, Susanna Widicus Weaver

III.31. Experimental studies of the formation of molecular hydrogen through superhydrogenation of PAHs

Emil E. Friis, John D. Thrower, Bjarke Jørgensen, Saoud Baouche, Alan Luntz, Liv Hornekær

III.32. Infrared spectroscopy of $\mathrm{D}_{2} \mathrm{O}$ in amorphous and crystalline water environments

Óscar Gálvez, Belén Maté, Rafael Escribano, Víctor J. Herrero

III.33. Electronic transitions of protonated PAHs in $6 \mathrm{~K}$ neon matrices

Iryna Garkusha, Jan Fulara, Adam Nagy, University of Basel, Sonia M.

Erattupuzha Joseph, John P. Maier

III.34. Modeling the formation of interstellar $\mathrm{CO}_{2}, \mathrm{CO}$ and water ice Robin Garrod, Tyler Pauly 
III.35. Modeling complex molecular chemistry in astrophysically-relevant laboratory ices

Robin Garrod, Karin Oberg

III.36. Sulfur and carbon chemistries driven by turbulent dissipation in the diffuse ISM

Benjamin Godard, Edith Falgarone, Guillaume Pineau des Forêts, Maryvonne Gerin, Massimo De Luca

III.37. The influence of cosmic rays on the 3.4 microns interstellar absorption band

Marie Godard, Géraldine Féraud, Marin Chabot, Thomas Pino, Rosario Brunetto, Yvain Carpentier, Jean Duprat, Cécile Engrand, Philippe

Bréchignac, Louis d'Hendecourt, Emmanuel Dartois

III.38. Visible spectra of titanium dioxide

Varun Gupta, Ramya Nagarajan, John P. Maier, Xiujuan Zhuang, Anh Le, Timothy C. Steimle

III.39. Cavity ring down spectroscopy of molecular ions in the 3 micron region

Joseph Guss, Harald Verbraak, Harold Linnartz

III.40. Diffusion mechanism of hydrogen and deuterium atoms and the spin temperature of molecules on the surface of water ice at $\mathbf{8 - 1 5} \mathrm{K}$

Tetsuya Hama, Naoki Watanabe, Yuki Kimura, Akira Kouchi, Takeshi Chigai, Valerio Pirronello

III.41. Ammonium and formate ions in interstellar ice analogues. Ice morphology and the elusive $6.85 \mu \mathrm{m}$ band

Víctor. J. Herrero, Belén Maté, Óscar Gálvez, Delia Fernández-Torre, Miguel

A. Moreno, Rafael Escribano

III.42. FTIR measurements of ammonia formation by the successive $\mathrm{H}$-atom addition to $\mathrm{N}$-atom in an $\mathrm{N}_{2}$ matrix at $10 \mathrm{~K}$

Hiroshi Hidaka, Motohiro Watanabe, Akira Kouchi, Naoki Watanabe

III.43. Formation routes of interstellar molecules through surface reactions at low temperature

Sergio Ioppolo, Gleb Fedoseev, Thanja Lamberts, Herma M. Cuppen, Claire Romanzin, Ewine F. van Dishoeck, Harold Linnartz

III.44. MATRIICES - Mass Analytical Tool for Reactions in Interstellar ICES

Karoliina Isokoski, Jean-Baptiste Bossa, Harold Linnartz

III.45. Photoprocessing of ice containing $\mathrm{H}_{2} \mathrm{~S}$ in circumstellar icy grain mantles

Antonio Jiménez-Escobar, Guillermo M. Muñoz Caro 
III.46. Solid-state spectroscopy: silicates \& carbonaceous grains Cornelia Jäger, Harald Mutschke, Thomas Henning, Friedrich Huisken

III.47. Associative detachment reaction $\mathrm{H}-+\mathrm{H}$ : Ion trap study at 10-100 K

Pavol Jusko, Štěpán Roučka, Illia Zymak, Dmytro Mulin, Sergii Opanasiuk, Radek Plašil, Dieter Gerlich, Juraj Glosík

III.48. UV irradiation of hydrogenated amorphous carbon (HAC) as a carrier candidate of the interstellar UV bump at $4.6 \mu \mathrm{m}^{-1}$

K. Gadallah, H. Mutschke, C. Jäger

III.49. Pyrolysis of simple chiral aromatic alcohols. Survivability and preservation of chirality on minerals of astrophysical interest: a case study

Yeghis Keheyan

III.50. Protonation of molecular oxygen probed in a cold 22-pole ion trap

Lars Kluge, I. Physikalisches Institut, Universität zu Köln, Sabrina Gärtner, I. Physikalisches Institut, Universität zu Köln, Sandra Brünken, I. Physikalisches Institut, Universität zu Köln, Oskar Asvany, I. Physikalisches Institut,

Universität zu Köln, Stephan Schlemmer, I. Physikalisches Institut, Universität zu Köln

III.51. Laboratory rotational spectrum of singly ${ }^{13} \mathrm{C}$-substituted dimethyl ether up to $1.5 \mathrm{THz}$ and interstellar detection of ${ }^{13} \mathrm{CH}_{3} \mathrm{O}^{12} \mathrm{CH}_{3}$ - a fruitful interplay between laboratory work and inter

Monika Koerber, Suzanne Bisschop, Christian Endres, Frank Lewen, Stephan Schlemmer,

III.52. Laboratory and observational studies of methyl ethyl ketone Jay A. Kroll, Steven Shipman, Susanna L. Widicus Weaver

III.53. The distributions of complex organic molecules in the Orion KL molecular core

Yi-Jehng Kuan, Yu-Sen Hsu, Steven B. Charnley, Kuo-Song Wang

III.54. Methanol photodissociation and its effects on complex chemistry in the ISM

Jacob Laas, Susanna Widicus Weaver

III.55. The revised version of class I methanol maser catalog G.M. Larionov, I.D. Litovchenko, I.E. Val'tts, A.V. Alakoz,

III.56. Atom - $H_{2}$ reactions at very low temperatures and astrophysical implications

Sébastien D. Le Picard, Meryem Tizniti, Coralie Berteloite, André Canosa, Ian R. Sims 
III.57. Competing mechanisms in molecular hydrogen formation on silicates in conditions relevant to the interstellar medium (up to $70 \mathrm{~K}$ )

Jean Louis Lemaire, Gianfranco Vidali, Saoud Baouche, Mourad Chehrouri, Henda Chaabouni, Hakima Mokrane

III.58. Applying the Meudon PDR code on dense structures from MHD simulations of the ISM

Francois Levrier, Franck Le Petit, Patrick Hennebelle, Pierre Lesaffre, Maryvonne Gerin, Edith Falgarone

III.59. Effects of reagent rotation and vibration on $\mathrm{H}+\mathrm{OH}(\mathrm{v}, \mathrm{j})-\mathrm{i}$ $\mathbf{O}+\mathbf{H}_{2}$

X. Li, Leiden Observatory, E. F. van Dishoeck, Leiden Observatory, M. C. van Hemert, Leiden Institute of Chemistry, C. Arasa

III.60. State-specific rate coefficients for dissociation of $\mathbf{H}_{2}(v, j)+$ $\mathbf{H}_{2}\left(v^{\prime}, j^{\prime}\right)$

Margot Mandy

III.61. An infrared study of glycine in astrophysical ices.

Belén Maté, Yamilet Rodríguez-Lazcano, Óscar Gálvez, Isabel Tanarro, Rafael Escribano

III.62. Residual gas analysis of samples formed from the UV irradiation of astrophysical ice analogs

Christopher K. Materese, Michel Nuevo, Scott A. Sandford

III.63. Progress towards the terahertz rotational spectrum of $\mathbf{H 5}+$ and its isotopologues

Brett A. McGuire, Yimin Wang, Joel Bowman, Susanna L. Widicus Weaver

III.64. Molecular hydrogen formation on aromatic carbon structures Vito Mennella, Liv Hornekaer, John Thrower, Mario Accolla

III.65. A solid state physics approach to the interaction between organic molecules and interstellar dust grains: $\left(C_{60}\right)$ on $\mathrm{SiC}$ Pablo Merino, Jose Angel Martin-Gago, Jose Cernicharo

III.66. PAH bombardment by energetic particles: models and astrophysical implications

Elisabetta Micelotta, Anthony Jones, Alexander Tielens

III.67. Effects of $\mathbf{H}_{2}$ coating of grains on depletion Oscar Morata, Tatsuhiko Hasegawa

III.68. Contrasting [CII], methylidynium ion $\mathrm{CH}+$, and methanol physical and chemical tracers around Orion KL

Pat Morris, John Pearson, David Neufeld, Harschal Gupta, and the HEXOS team 
III.69. QUB low energy ion-ices irradiation experiment Andra Muntean, Tom Field, Adam. Hunniford, Bob McCullough, Jorge Konanoff, Tom Millar

III.70. Electronic absorption spectra of mass-selected hydrocarbon cations in solid neon: $\mathrm{C}_{n} \mathbf{H}_{4}^{+}(n=5-8,10,12)$

Adam Nagy, Jan Fulara, Iryna Garkusha, John P. Maier

III.71. Electron irradiation of interstellar ice analogues

B. G. Nair, N. J. Mason

III.72. Formation of nucleobases and other prebiotic species from the UV irradiation of pyrimidine in astrophysical ices

Michel Nuevo, Scott A. Sandford, Stefanie N. Milam, Christopher K. Materese, Jamie E. Elsila, Jason P. Dworkin

III.73. Sequential hydrogenation of molecular oxygen on cold surfaces: reaction kinetics and structure of formed $\mathrm{H}_{2} \mathrm{O}$ ice. Yasuhiro Oba, Naoya Miyauchi, Hiroshi Hidaka, Takeshi Chigai, Naoki Watanabe, Akira Kouchi

III.74. Neutral radical-molecule reactions $\mathrm{CO}+\mathrm{OH}$ on cold interstellar ices

Yasuhiro Oba, Naoki Watanabe, Akira Kouchi, Tetsuya Hama, Valerio Pirronello

III.75. Formation of $\mathrm{CO}_{2}$ and OCS after cosmic ion irradiation of icy grain mantles

M. Elisabetta Palumbo, Giuseppe Baratta, Daniele Fulvio, Mario Garozzo, Sergio Ioppolo, Zuzana Kanuchova, Giuseppe Leto, Ivana Sangiorgio, Giovanni Strazzulla

III.76. About the role of ice in the reconstruction of ice F. Pauzat, P. Redondo, A. Markovits, Y. Ellinger

III.77. A submillimeter study of $\mathrm{CH}$ in Orion $\mathrm{KL}$ John C Pearson, Harshal Gupta, Patrick Morris, Maryvonne Gerin, Edwin Bergin

III.78. A hybrid method of moment equations and rate equations to modeling gas-grain chemistry

Yezhe Pei, Eric Herbst

III.79. An approach to global rovibrational analysis based on anharmonic ladder operators: Application to hydrogen selenide and hydrogen sulfide

F. Pérez Bernal, M. Carvajal, O. Álvarez-Bajo 
III.80. Secondary fragmentation routes of glycine in ice under irradiation

A. Pernet, J. Pilmé, Y. Ellinger,

III.81. Near-infrared to visible spectral lines of cold $\mathbf{H}_{3}^{+}$ Annemieke Petrignani, Max Berg, Dennis Bing, Florian Grussie, Andreas Wolf

III.82. Possible evidence for crystallization of astrophysical ice analogs by heavy and energetic cosmic rays

Sergio Pilling, Eduardo Seperuelo Duarte, Enio F. da Silveira, Hermann Rothard, Alicja Domaracka, Philippe Boduch

III.83. Formation of unsaturated hydrocarbons by cosmic ray analogs in interstellar ices

Sergio Pilling, Diana P. P. Andrade, Enio F. da Silveira, Hermann Rothard, Alicja Domaracka, Philippe Boduch

III.84. Chiral molecules in the ISM: the best candidates J. Pilmé, G. Marloie, M. Lattelais, F. Pauzat, Y. Ellinger

III.85. An FTIR study on the catalytic effect of water molecules on the reaction of $\mathrm{CO}$ successive hydrogenation at 3 and $10 \mathrm{~K}$ Claire Pirim, Lahouari Krim

III.86. Surface science studies of ethene containing model interstellar ices

Fabrizio Puletti, Mark Whelan, Wendy A. Brown

III.87. Reaching the line confusion limit: new spectral analysis software and its application to a molecular line survey of Orion-KL

Mary L. Radhuber, Matthew C. Sumner, Jay A. Kroll, Jonas Zmuidzinas, Georey A. Blake, Susanna L. Widicus Weaver

III.88. Chemical and physical conditions at the disk-halo interaction places in the Galactic center region

Denise Riquelme, M. Aranzazu Amo-Baladron, Jesus Martin-Pintado, Rainer Mauersberger, Sergio Martin, Leonardo Bronfman

III.89. $\mathrm{H}_{2}$ formation pumping signatures: a modellist's view Evelyne Roueff, Jacques Le Bourlot, Franck Le Petit, Simon Casassus

III.90. Ab initio calculations for the spectral analysis of dimethyl ether $\left(\mathrm{CH}_{3} \mathrm{OCH}_{3}\right)$ and their isotopologues

M.L. Senent, M. Villa, R. Domínguez-Gómez, O. Álvarez-Bajo, M. Carvajal

III.91. H atom diffusion on amorphous ice at low temperature

Bethmini Senevirathne, Gunnar Nyman, S. Andersson 
III.92. A Spitzer survey of an isolated globule: DC314.8-5.1

S.S. Shenoy, D.C.B. Whittet, Y.J. Pendleton, C. Boersma, L.J. Allamandola, D. Horne, P.A. Mayeur

III.93. Deuterium fractionation of massive star forming clumps in infrared dark clouds

Yu-Nung Su, Sheng-Yuan Liu, Vivien H.-R. Chen, Qizhou Zhang

III.94. Dissociative electron attachment to hydrocarbons. A laboratory study

E. Szymanska, N. J. Mason

III.95. A theoretical study of the electronic dissociative recombination of an ion of astrophysical interest : $\mathbf{N}_{2} \mathbf{H}+$ Dahbia Talbi, David Kashinski, Peet Hickman

III.96. SUCCESS: A SUbmm Catalogue of Circumstellar Envelope of StarS with Herschel/HIFI

David Teyssier, Javier Alcolea, Valentin Bujarrabal, Arantxa Castro-Carrizo, Jose Cernicharo, Pedro Garcia-Lario, Anthony Marston, Hans Olofsson, Christophe Risacher, Fredrik Schoeier, Eva Verdugo

III.97. Kinetic studies on low-temperature solid-state reactions in interstellar ices

Patrice Theule, Fabrice Duvernay, Florent Mispelaer, Vassilissa Vinogradoff, Jean-Baptiste Bossa, Fabien Borget, Gregoire Danger, Thierry Chiavassa

III.98. Infrared spectroscopy of carbon- and carbon-silicon clusters Sven Thorwirth, Jürgen Krieg, Volker Lutter, Imke Gottbehüt, Stephan Schlemmer, Thomas F. Giesen

III.99. Laboratory studies of astronomically relevant molecules harboring second-row elements

Sven Thorwirth, Valerio Lattanzi, Leonie A. Mück, Dewayne T. Halfen, Sandra Brünken, Carl A. Gottlieb, Patrick Thaddeus, Lucy M. Ziurys, Jürgen Gauss, Michael C. McCarthy

III.100. Isotope effect on charge transfer in collisions of $\mathbf{H}$ with $\mathrm{He}^{+}$ and $\mathbf{H e}^{2+}$

Nathalie Vaeck, Jérôme Loreau, Sergey Ryabchenko

III.101. Recent development of the photoionization / PDR code Cloudy

P.A.M. van Hoof, R.L. Porter, G.J. Ferland

III.102. Formation of water on dust grains

Gianfranco Vidali, Dapeng Jing, Jiao He, Paul Frank, John Brucato, Antonio De Sio, Lorenzo Tozzetti 
III.103. Chemical evolution of the interstellar ice analogs: HMT formation mechanism

Vassilissa Vinogradoff, Fabrice Duvernay, Grégoire Danger, Patrice Theulé, Fabien Borget, T. Chiavassa

III.104. Photodissociation and radiative stabilization of $\mathrm{HeH}^{+}$in its $a$ and $b^{3} \Sigma^{+}$states

Stéphane Vranckx, Jérôme Loreau, Michèle Desouter-Lecomte, Nathalie Vaeck

III.105. The KInetic Database for Astrochemistry (KIDA)

V. Wakelam and the KIDA team

III.106. New molecules in hydrogen precipitates

Mark Walker, Leaf Lin, Andrew Gilbert, Peter Gill

III.107. Deuterated glycoaldehyde: laboratory measurements, analysis and proposed astrophysical research

Aurélia Bouchez, Adam Walters, Laurent Margulés, Roman Motiyenko, Jean-Claude Guillemin, Sandrine Bottinelli, Cecilia Ceccarelli, Claudine Kahane

III.108. Laboratory measurements and analysis for two molecules of astrophysical interest: ${ }^{13} \mathrm{C}$-ethanol and $n$-butyl cyanide

Adam Walters, Matthias Ordu, Aurélia Bouchez, Holger Müller, Marc Nuñez, Frank Lewen, Sandrine Bottinelli, Stephan Schlemmer

III.109. Isolation of $\mathrm{OH}$ radicals in water ice and study of their reactivity

Emilie-Laure Zins, Prasad Joshi, Lahouari Krim,

III.110. T BASECOL and VAMDC: A new way to access and manipulate molecular collisional data for interstellar applications

F. Daniel, M. L. Dubernet, M. Doronin, L. Nenadovic, J. Bureau

III.111. The chemistry of AGB stars at low metallicity

Paul Woods, Ciska Kemper, Martin Cordiner

III.112. Amino Acids and Sugars in the Gas Phase: Microwave Data for Astrochemistry

Santiago Mata, Carlos Cabezas, Marcelino Varela, Isabel Peña, Cristobal

Pérez, Susana Blanco, María E. Sanz, Juan C. López, José L. Alonso

III.113. Commissioning and Science Verification of ALMA

R. Mauersberger, E. Villard, A. B. Peck, S. Asayama, D. Barkats, P. Calisse,S. Corder, J. Cortes, P. Cortes, I. de Gregorio, W. R. F. Dent, E. Fomalont, D. Fulla, D. Garcia-Appadoo, D. Gunawan, A. Hales, R.E. Hills, T. Kamazaki, L. Knee, R. Kneissl, S. Komugi, S. Leon, R. Lucas, A. Lundgren, G. Marconi, S. Matsushita, P. Planesas, M. Rawlings, A. Richards, T. Sawada, R. Simon, K. Tachihara, T. van Kempen, T. Wiklind 
III.114. The ORGANIC Experiment on the ISS EXPOSE-R

K. Bryson, Z. Peeters, F. Salama, B. Foing, P. Ehrenfreund, E. Jessberger, A. Bischoff, M. Breitfellner, W. Schmidt

III.115. Experimental results obtained with the Interstellar Astrochemistry Chamber (ISAC) for the simulation of ice processes

G. M. Muñoz Caro, A. Jimenez Escobar, G.A. Cruz Diaz, A. Ciaravella, C. Cecchi-Pestellini

III.116. Composition of the grain mantle: A Monte Carlo Study

A. Das, S. K. Chakrabarti

III.117. Chemical Evolution around star forming region: A time dependent study

L. Majumdar, A. Das, S.K. Chakrabarti, S. Chakrabarti 